\title{
Fourier spectral method for the modified Swift-Hohenberg equation
}

\author{
Xiaopeng Zhao ${ }^{1 *}$, Bo Liu' ${ }^{1}$ Peng Zhang ${ }^{2}$, Wenyu Zhang ${ }^{1}$ and Fengnan Liu'
}

"Correspondence: zxp032@126.com

'College of Mathematics, Jilin

University, Changchun, 130012, China

Full list of author information is available at the end of the article

\section{Abstract}

In this paper, we consider the Fourier spectral method for numerically solving the modified Swift-Hohenberg equation. The semi-discrete and fully discrete schemes are established. Moreover, the existence, uniqueness and the optimal error bound are also considered.

\section{Introduction}

In [1], Doelman et al. studied the modified Swift-Hohenberg equation

$$
u_{t}=-k(1+\Delta)^{2} u+\mu u-b|\nabla u|^{2}-u^{3} .
$$

Setting $a=k-\mu$, considering (1) in 1D case, we find that

$$
u_{t}+k u_{x x x x}+2 k u_{x x}+a u+b\left|u_{x}\right|^{2}+u^{3}=0, \quad(x, t) \in(0,1) \times(0, T) .
$$

On the basis of physical considerations, as usual, Eq. (2) is supplemented with the following boundary value conditions:

$$
u(x, t)=u_{x x}(x, t)=0, \quad x=0,1
$$

and the initial condition

$$
u(x, 0)=u_{0}(x), \quad x \in(0,1)
$$

where $k>0$ and $a, b$ are constants. $u_{0}(x)$ is a given function from a suitable phase space.

The Swift-Hohenberg equation is one of the universal equations used in the description of pattern formation in spatially extended dissipative systems (see [2]), which arise in the study of convective hydrodynamics [3], plasma confinement in toroidal devices [4], viscous film flow and bifurcating solutions of the Navier-Stokes [5]. Note that the usual Swift-Hohenberg equation [3] is recovered for $b=0$. The additional term $b\left|u_{x}\right|^{2}$, reminiscent of the Kuramoto-Sivashinsky equation, which arises in the study of various pattern formation phenomena involving some kind of phase turbulence or phase transition (see [6-8]), breaks the symmetry $u \rightarrow-u$.

\section{Springer}

(0) 2013 Zhao et al.; licensee Springer. This is an Open Access article distributed under the terms of the Creative Commons Attribution License (http://creativecommons.org/licenses/by/2.0), which permits unrestricted use, distribution, and reproduction in any medium, provided the original work is properly cited. 
During the past years, many authors have paid much attention to the Swift-Hohenberg equation (see, e.g., $[3,9,10]$ ). However, only a few people have been devoted to the modified Swift-Hohenberg equation. It was Doelman et al. [1] who first studied the modified Swift-Hohenberg equation for a pattern formation system with two unbounded spatial directions that are near the onset to instability. Polat [7] also considered the modified Swift-Hohenberg equation. In his paper, the existence of a global attractor is proved for the modified Swift-Hohenberg equation as (2)-(4). Recently, Song et al. [2] studied the long time behavior for the modified Swift-Hohenberg equation in an $H^{k}(k \geq 0)$ space. By using an iteration procedure, regularity estimates for the linear semigroups and a classical existence theorem of a global attractor, they proved that problem (2)-(4) possesses a global attractor in the Sobolev space $H^{k}$ for all $k \geq 0$, which attracts any bounded subset of $H^{k}(\Omega)$ in the $H^{k}$-norm.

The spectral methods are essentially discretization methods for the approximate solution of partial differential equations. They have the natural advantage in keeping the physical properties of primitive problems. During the past years, many papers have already been published to study the spectral methods, for example, [11-14]. However, for the other boundary condition, can we also use the Fourier spectral method? The answer is 'Yes'. Choose a good finite dimensional subspace $S_{N}$ (here, we set $S_{N}=\operatorname{span}\{\sin k \pi x ; k=$ $0,1, \ldots, N\}$ ), we can also have the basic results for the orthogonal projecting operator $P_{N}$. In this paper, we consider the Fourier spectral method for the modified Swift-Hohenberg equation. The existence of a solution locally in time is proved by the standard Picard iteration, global existence results are obtained by proving a priori estimate for the appropriate norms of $u(x, t)$. Adjusted to our needs, the results are given in the following form.

Theorem 1.1 Assume that $u_{0} \in H_{E}^{2}(0,1)=\left\{v ; v \in H^{2}(0,1), v(0, t)=v(1, t)=0\right\}$ and $b^{2} \leq 8 k$, then there exists a unique global solution $u(x, t)$ of the problem (2)-(4) for all $T \geq 0$ such that

$$
u(x, t) \in L^{\infty}\left(0, T ; H_{E}^{2}(0,1)\right) \cap L^{2}\left(0, T ; H^{4}(0,1)\right) .
$$

Furthermore, it satisfies

$$
\begin{aligned}
& \left(\frac{\partial u}{\partial t}, v\right)+k\left(u_{x x}, v_{x x}\right)-2 k\left(u_{x}, v_{x}\right)+\gamma(u, v) \\
& +b\left(\left|u_{x}\right|^{2}, v\right)+\left(u^{3}, v\right)=0, \quad \forall v \in H_{E}^{2}(0,1), \\
& (u(\cdot, 0), v)=\left(u_{0}, v\right), \quad \forall v \in H_{E}^{2}(0,1) .
\end{aligned}
$$

This paper is organized as follows. In the next section, we consider a semi-discrete Fourier spectral approximation, prove its existence and uniqueness of the numerical solution and derive the error bound. In Section 3, we consider the full-discrete approximation for problem (2)-(4). Furthermore, we prove convergence to the solution of the associated continuous problem. In the last section, some numerical experiments which confirm our results are performed.

Throughout this paper, we denote $L^{2}, L^{p}, L^{\infty}, H^{k}$ norm in $\Omega$ simply by $\|\cdot\|,\|\cdot\|_{p},\|\cdot\|_{\infty}$ and $\|\cdot\|_{H^{k}}$. 


\section{Semi-discrete approximation}

In this section, we consider the semi-discrete approximation for problem (2)-(4). First of all, we recall some basic results on the Fourier spectral method which will be used throughout this paper. For any integer $N>0$, introduce the finite dimensional subspace of $H_{E}^{2}(0,1)$

$$
S_{N}=\operatorname{span}\{\sin k \pi x ; k=1, \ldots, N\} .
$$

Let $P_{N}: L^{2}(0,1) \rightarrow S_{N}$ be an orthogonal projecting operator which satisfies

$$
\left(u-P_{N} u, v\right)=0, \quad \forall v \in S_{N} .
$$

For the operator $P_{N}$, we have the following result (see $\left.[13,15]\right)$ :

(B1) $P_{N}$ commutes with derivation on $H_{E}^{2}(0,1)$, i.e.,

$$
P_{N} u_{x x}=\left(P_{N} u\right)_{x x}, \quad \forall u \in H_{E}^{2}(0,1) .
$$

Using the same method as $[15,16]$, we can obtain the following result (B2) for problem (2)-(4):

(B2) For any real $0 \leq \mu \leq 2$, there is a constant $c$ such that

$$
\left\|u-P_{N} u\right\|_{\mu} \leq c N^{\mu-2}\left\|u_{x x}\right\|, \quad \forall u \in H_{E}^{2}(0,1) .
$$

Define the Fourier spectral approximation: Find $u_{N}(t)=\sum_{j=1}^{N} a_{j}(t) \cos j \pi x \in S_{N}$ such that

$$
\begin{aligned}
& \left(\frac{\partial u_{N}}{\partial t}, v_{N}\right)+k\left(u_{N x x}, v_{N x x}\right)+2 k\left(u_{N}, v_{N x x}\right)+a\left(u_{N}, v_{N}\right) \\
& \quad+b\left(\left|u_{N x}\right|^{2}, v_{N}\right)+\left(u_{N}^{3}, v_{N}\right)=0, \quad \forall v_{N} \in S_{N}
\end{aligned}
$$

for all $T \geq 0$ with $u_{N}(0)=P_{N} u_{0}$.

Now, we are going to establish the existence, uniqueness etc. of the Fourier spectral approximation solution $u_{N}(t)$ for all $T \geq 0$.

Lemma 2.1 Let $u_{0} \in L^{2}(0,1)$ and $b^{2} \leq 8 k$, then problem (7) has a unique solution $u_{N}(t)$ satisfying the following inequalities:

$$
\left\|u_{N}(t)\right\|^{2} \leq c_{1}\left\|u_{0}\right\|^{2}, \quad \int_{0}^{T}\left\|u_{N x x}(\tau)\right\|^{2} d \tau \leq c_{1}^{\prime}\left\|u_{0}\right\|^{2}
$$

where $c_{1}=e^{(|a|+2) T}$ and $c_{1}^{\prime}=\frac{8(|a|+2) T c_{1}+8}{8 k-b^{2}}$ for all $T \geq 0$.

Proof Set $v_{N}=\cos j \pi x$ in (7) for each $j(1 \leq j \leq N)$ to obtain

$$
\frac{d}{d t} a_{j}(t)=f_{j}\left(a_{1}(t), a_{2}(t), \ldots, a_{N}(t)\right), \quad j=1,2, \ldots, N
$$


where all $f_{j}: \mathbb{R}^{N} \rightarrow \mathbb{R}(1 \leq j \leq N)$ are smooth and locally Lipschitz continuous. Noticing that $u_{N}(0)=P_{N} u_{0}$, then

$$
a_{j}(0)=\left(u_{0}, \cos j \pi x\right), \quad j=1,2, \ldots, N .
$$

Using the theory of initial-value problems of the ordinary differential equations, there is a time $T_{N}>0$ such that the initial-value problem (9)-(10) has a unique smooth solution $\left(a_{1}(t), \ldots, a_{N}(t)\right)$ for $t \in\left[0, T_{N}\right]$.

Setting $v_{N}=u_{N}$ in (7), we have

$$
\frac{1}{2} \frac{d}{d t}\left\|u_{N}\right\|^{2}+k\left\|u_{N x x}\right\|^{2}+\left\|u_{N}\right\|_{4}^{4} \leq|a|\left\|u_{N}\right\|^{2}+2 k\left\|u_{N x}\right\|^{2}+|b|\left(\left|u_{N x}\right|^{2}, u_{N}\right) .
$$

Noticing that

$$
|b|\left(\left|u_{N x}\right|^{2}, u_{N}\right)=-\frac{|b|}{2}\left(u_{N}^{2}, u_{N x x}\right) \leq\left\|u_{N}\right\|_{4}^{4}+\frac{b^{2}}{16}\left\|u_{N x x}\right\|^{2}
$$

and

$$
2 k\left\|u_{N x}\right\|^{2}=-2 k\left(u_{N}, u_{N x x}\right) \leq \frac{k}{2}\left\|u_{N x x}\right\|^{2}+2 k\left\|u_{N}\right\|^{2} .
$$

Summing up, we get

$$
\frac{d}{d t}\left\|u_{N}\right\|^{2}+\left(k-\frac{b^{2}}{8}\right)\left\|u_{N x x}\right\|^{2} \leq 2(|a|+2 k)\left\|u_{N}\right\|^{2} .
$$

Using Gronwall's inequality, we deduce that

$$
\left\|u_{N}\right\|^{2} \leq e^{2(|a|+2 k) t}\left\|u_{N}(0)\right\|^{2} \leq e^{2(|a|+2 k) T}\left\|u_{0}\right\|^{2}=c_{1}\left\|u_{0}\right\|^{2} .
$$

Integrating (12) from 0 to $t$, we derive that

$$
\left\|u_{N}\right\|^{2}-\left\|u_{N}(0)\right\|^{2}+\left(k-\frac{b^{2}}{8}\right) \int_{0}^{T}\left\|u_{N x x}\right\|^{2} d t \leq(2|a|+4 k) \int_{0}^{T}\left\|u_{N}\right\|^{2} d t .
$$

Hence

$$
\left(k-\frac{b^{2}}{8}\right) \int_{0}^{T}\left\|u_{N x x}\right\|^{2} d t \leq 2(|a|+2 k) c_{1} T\left\|u_{0}\right\|^{2}+\left\|u_{0}\right\|^{2} .
$$

From the above inequality, we obtain the second inequality of (8) immediately. Therefore, Lemma 2.1 is proved.

Lemma 2.2 Let $u_{0} \in H_{0}^{1}(0,1)$ and $b^{2} \leq 8 k$, then the solution $u_{N}(t)$ of problem (7) satisfying

$$
\left\|u_{N x}(t)\right\|^{2} \leq c_{2}, \quad \int_{0}^{T}\left\|u_{n x x x}(\tau)\right\|^{2} d \tau \leq c_{2}^{\prime}
$$

for all $T \geq 0$, where $c_{2}$ and $c_{2}^{\prime}$ are positive constants depending only on $k, a, b, T$ and $\left\|u_{0}\right\|_{H^{1}}$. 
Proof Setting $v_{N}=u_{N x x}$ in (7), we obtain

$$
\frac{1}{2} \frac{d}{d t}\left\|u_{N x}\right\|^{2}+k\left\|u_{N x x x}\right\|^{2}+a\left\|u_{N x}\right\|^{2}=2 k\left\|u_{N x x}\right\|^{2}+b\left(\left|u_{N x}\right|^{2}, u_{N x x}\right)+\left(u_{N}^{3}, u_{N x x}\right)
$$

Notice that

$$
\left(u_{N}^{3}, u_{N x x}\right)=-\left(3 u_{N}^{2} u_{N x}, u_{N x}\right)=-3\left\|u_{N} u_{N x}\right\|^{2} \leq 0
$$

and

$$
2 k\left\|u_{N x x}\right\|^{2}=-2 k\left(u_{N x}, u_{N x x x}\right) \leq \frac{k}{4}\left\|u_{N x x x}\right\|^{2}+4\left\|u_{N x}\right\|^{2} .
$$

On the other hand, by Nirenberg's inequality, we have

$$
\left\|u_{N x}\right\|_{4} \leq C\left\|u_{N x x x}\right\|^{\frac{5}{12}}\left\|u_{N}\right\|^{\frac{7}{12}}
$$

where $C$ is a positive constant independent of $N$. Hence

$$
\begin{aligned}
|b|\left(\left|u_{N x}\right|^{2}, u_{N x x}\right) & \leq \frac{|b|}{2}\left\|u_{N x x}\right\|^{2}+\frac{|b|}{2}\left\|u_{N x}\right\|_{4}^{4} \\
& \leq \frac{k}{8}\left\|u_{N x x x}\right\|^{2}+\frac{|b|^{2}}{2 k}\left\|u_{N x}\right\|^{2}+C^{4}\left\|u_{N x x x}\right\|^{\frac{5}{3}}\left\|u_{N}\right\|^{\frac{7}{3}} \\
& \leq \frac{k}{4}\left\|u_{N x x x}\right\|^{2}+\frac{|b|^{2}}{2 k}\left\|u_{N x}\right\|^{2}+c\left(\left\|u_{N}\right\|\right) \\
& \leq \frac{k}{4}\left\|u_{N x x x}\right\|^{2}+\frac{|b|^{2}}{2 k}\left\|u_{N x}\right\|^{2}+c\left(a, k, T,\left\|u_{0}\right\|\right) .
\end{aligned}
$$

Summing up, we get

$$
\frac{d}{d t}\left\|u_{N x}\right\|^{2}+k\left\|u_{N x x x}\right\|^{2} \leq\left(2|a|+8+\frac{b^{2}}{k}\right)\left\|u_{N x}\right\|^{2}+2 c\left(a, k, T,\left\|u_{0}\right\|\right) .
$$

Using Gronwall's inequality, we immediately obtain

$$
\begin{aligned}
\left\|u_{N x}\right\|^{2} & \leq e^{\left(2|a|+8+\frac{b^{2}}{k}\right) t}\left\|u_{N x}(0)\right\|^{2}+\frac{2 k c\left(a, k, T,\left\|u_{0}\right\|\right)}{2|a| k+b^{2}+8 k} \\
& \leq e^{\left(2|a|+8+\frac{b^{2}}{k}\right) T}\left\|u_{x 0}\right\|^{2}+\frac{2 k c\left(a, k, T,\left\|u_{0}\right\|\right)}{2|a| k+b^{2}+8 k} \leq c_{2}\left(k, a, b, T,\left\|u_{0}\right\|_{H^{1}}\right) .
\end{aligned}
$$

Integrating (16) from 0 to $t$, we obtain

$$
\begin{aligned}
\int_{0}^{T}\left\|u_{N x x x}(\tau)\right\|^{2} d \tau & \leq \frac{1}{k}\left(\left(2|a|+8+\frac{b^{2}}{k}\right) c_{2} T+2 c T+\left\|u_{x 0}\right\|^{2}\right) \\
& =c_{2}^{\prime}\left(k, a, b, T,\left\|u_{0}\right\|_{H^{1}}\right) .
\end{aligned}
$$

Then Lemma 2.2 is proved. 
Lemma 2.3 Let $u_{0} \in H_{E}^{2}(0,1)$ and $b^{2} \leq 8 k$, then the solution $u_{N}(t)$ of problem $(7)$, satisfying

$$
\left\|u_{N x x}(t)\right\|^{2} \leq c_{3}, \quad \int_{0}^{T}\left\|u_{N x x x x}(\tau)\right\|^{2} d \tau \leq c_{3}^{\prime}
$$

for all $T \geq 0$, where $c_{3}$ and $c_{3}^{\prime}$ are positive constants, depending only on $k, a, b, T$ and $\left\|u_{0}\right\|_{H^{2}}$.

Proof Setting $v_{N}=u_{N x x x x}$ in (7), we obtain

$$
\begin{aligned}
& \frac{1}{2} \frac{d}{d t}\left\|u_{N x x}\right\|^{2}+k\left\|u_{N x x x x}\right\|^{2} \\
& \quad=2\left\|u_{N x x x}\right\|^{2}-a\left\|u_{N x x}\right\|^{2}-b\left(\left|u_{N x}\right|^{2}, u_{N x x x x}\right)-\left(u_{N}^{3}, u_{N x x x x}\right) .
\end{aligned}
$$

Using Nirenberg's inequality, we obtain

$$
\left\|u_{N}\right\|_{6} \leq C\left\|u_{N x x x x}\right\|^{\frac{1}{12}}\left\|u_{N}\right\|^{\frac{11}{12}}, \quad\left\|u_{N x}\right\|_{4} \leq C\left\|u_{N x x x x}\right\|^{\frac{1}{12}}\left\|u_{N x}\right\|^{\frac{11}{12}}
$$

where $C>0$ is a constant depending only on the domain. Therefore

$$
\begin{aligned}
|b|\left(\left|u_{N x}\right|^{2}, u_{N x x x x}\right) & \leq \frac{k}{10}\left\|u_{N x x x x}\right\|^{2}+\frac{5 b^{2}}{2 k}\left\|u_{N x}\right\|_{4}^{4} \\
& \leq \frac{k}{10}\left\|u_{N x x x x}\right\|^{2}+\frac{k}{10}\left\|u_{N x x x x}\right\|^{2}+c\left(a, k, b, T,\left\|u_{0}\right\|_{H^{1}}\right)
\end{aligned}
$$

and

$$
\begin{aligned}
\left(u_{N}^{3}, u_{N x x x x}\right) & \leq \frac{k}{10}\left\|u_{N x x x x}\right\|^{2}+\frac{5}{2 k}\left\|u_{N}\right\|_{6}^{6} \\
& \leq \frac{k}{10}\left\|u_{N x x x x}\right\|^{2}+\frac{k}{10}\left\|u_{N x x x x}\right\|^{2}+c\left(a, k, T,\left\|u_{0}\right\|\right) .
\end{aligned}
$$

On the other hand, we have

$$
2 k\left\|u_{N x x x}\right\|^{2}=-2 k\left(u_{N x x}, u_{N x x x x}\right) \leq \frac{k}{10}\left\|u_{N x x x x}\right\|^{2}+10\left\|u_{N x x}\right\|^{2} .
$$

Summing up, we get

$$
\frac{d}{d t}\left\|u_{N x x}\right\|^{2}+k\left\|u_{N x x x x}\right\|^{2} \leq(20+2|a|)\left\|u_{N x x}\right\|^{2}+4 c\left(a, k, b, T,\left\|u_{0}\right\|_{H^{1}}\right) .
$$

Using Gronwall's inequality, we have

$$
\begin{aligned}
\left\|u_{N x x}\right\|^{2} & \leq e^{(20+2|a|) t}\left\|u_{N x x}(0)\right\|^{2}+\frac{2 c\left(a, k, b, T,\left\|u_{0}\right\|_{H^{1}}\right)}{10+|a|} \\
& \leq e^{(20+2|a|) T}\left\|u_{x x 0}\right\|^{2}+\frac{2 c\left(a, k, b, T,\left\|u_{0}\right\|_{H^{1}}\right)}{10+|a|}=c_{3}\left(a, k, b, T,\left\|u_{x x 0}\right\|\right) .
\end{aligned}
$$


Integrating (19) from 0 to $t$, we obtain

$$
\begin{aligned}
\int_{0}^{T}\left\|u_{N x x x x}(\tau)\right\|^{2} d \tau & \leq \frac{1}{k}\left((20+2|a|) c_{3} T+4 T c\left(a, k, b, T,\left\|u_{0}\right\|_{H^{1}}\right)+\left\|u_{N x x}(0)\right\|^{2}\right) \\
& =c_{3}^{\prime}\left(k, a, b, T,\left\|u_{0}\right\|_{H^{2}}\right) .
\end{aligned}
$$

Therefore, Lemma 2.3 is proved.

Remark 2.1 Basing on the above Lemmas 2.1-2.3, we can get the $H^{2}$-norm estimate for problem (7). Then, by Sobolev's embedding theorem, we immediately conclude that

$$
\begin{aligned}
& \sup _{x \in[0,1]}\left|u_{N}(x, t)\right|=\left\|u_{N}(x, t)\right\|_{\infty} \leq c_{4}\left(k, a, b, T,\left\|u_{0}\right\|_{H^{1}}\right), \\
& \sup _{x \in[0,1]}\left|u_{N x}(x, t)\right|=\left\|u_{N x}(x, t)\right\|_{\infty} \leq c_{5}\left(k, a, b, T,\left\|u_{0}\right\|_{H^{2}}\right) .
\end{aligned}
$$

Now, we give the following theorem.

Theorem 2.1 Suppose that $u_{0} \in H_{E}^{2}(0,1)$ and $b^{2} \leq 8 k$. Suppose further that $u(x, t)$ is the solution of problem (2)-(4) and $u_{N}(x, t)$ is the solution of semi-discrete approximation (7). Then there exist a constant $c$ depending on $k, a, b, T$ and $\left\|u_{0}\right\|_{H^{2}}$ such that

$$
\left\|u(x, t)-u_{N}(x, t)\right\| \leq c\left(N^{-2}+\left\|u_{0}-u_{N}(0)\right\|\right) .
$$

Proof Denote $\eta_{N}=u(t)-P_{N} u(t)$ and $e_{N}=P_{N} u(t)-u_{N}(t)$. From (2) and (7), we get

$$
\begin{aligned}
& \left(e_{N t}, v_{N}\right)+k\left(e_{N x x}, v_{N x x}\right)-2 k\left(e_{N x}, v_{N x}\right)+a\left(e_{N}, v_{N}\right) \\
& \quad+b\left(\left|u_{x}\right|^{2}-\left|u_{N x}\right|^{2}, v_{N}\right)+\left(u^{3}-u_{N}^{3}, v_{N}\right)=0, \quad \forall v_{N} \in S_{N} .
\end{aligned}
$$

Set $v_{N}=e_{N}$ in (23), we derive that

$$
\frac{1}{2} \frac{d}{d t}\left\|e_{N}\right\|^{2}+k\left\|e_{N x x}\right\|^{2}=2 k\left\|e_{N x}\right\|^{2}-a\left\|e_{N}\right\|^{2}-b\left(\left|u_{x}\right|^{2}-\left|u_{N x}\right|^{2}, e_{N}\right)-\left(u^{3}-u_{N}^{3}, e_{N}\right)
$$

By Theorem 1.1, we have

$$
\sup _{x \in[0,1]}|u(x, t)| \leq c_{6}\left(k, a, b,\left\|u_{0}\right\|_{H^{1}}\right) .
$$

Then

$$
\begin{aligned}
& -\left(u^{3}-u_{N}^{3}, e_{N}\right) \\
& \quad=-\left(\left(e_{N}+\eta_{N}\right)\left(u^{2}+u u_{N}+u_{N}^{2}\right), e_{N}\right) \\
& \quad \leq \sup _{x \in[0,1]}\left(|u|^{2}+\left|u u_{N}\right|+\left|u_{N}\right|^{2}\right) \cdot\left(\left\|e_{N}\right\|^{2}+\left\|\eta_{N}\right\|\left\|e_{N}\right\|\right) \\
& \quad \leq\left(c_{4}^{2}+c_{4} c_{6}+c_{6}^{2}\right)\left(\left\|e_{N}\right\|^{2}+\frac{1}{2}\left\|e_{N}\right\|^{2}+\frac{1}{2}\left\|\eta_{N}\right\|^{2}\right) .
\end{aligned}
$$


By Theorem 1.1, we have

$$
\sup _{x \in[0,1]}\left|u_{x}(x, t)\right| \leq c_{7}\left(k, a, b,\left\|u_{0}\right\|_{H^{2}}\right), \quad\left\|u_{x x}(x, t)\right\|^{2} \leq c_{8}\left(k, a, b,\left\|u_{0}\right\|_{H^{2}}\right) .
$$

Using Sobolev's embedding theorem, we have

$$
\sup _{x \in[0,1]}\left|e_{N x}\right| \leq C^{\prime}\left\|e_{N}\right\|_{H^{2}} \leq C\left\|e_{N x x}\right\|
$$

where $C^{\prime}$ and $C$ are positive constants depending only on the domain. Then, using the method of integration by parts, we have

$$
\begin{aligned}
-b\left(\left|u_{x}\right|^{2}-\left|u_{N x}\right|^{2}, e_{N}\right)= & -b\left(\left(e_{N x}+\eta_{N x}\right)\left(u_{x}+u_{N x}\right), e_{N}\right) \\
= & b\left(\left(e_{N}+\eta_{N}\right)\left(u_{x}+u_{N x}\right), e_{N x}\right) \\
& +b\left(\left(e_{N}+\eta_{N}\right)\left(u_{x x}+u_{N x x}\right), e_{N}\right) .
\end{aligned}
$$

Hence, by (24)-(26) and Hölder's inequality, we get

$$
\begin{aligned}
-b & \left(\left|u_{x}\right|^{2}-\left|u_{N x}\right|^{2}, e_{N}\right) \\
\leq & |b| \sup _{x \in[0,1]}\left|u_{x}+u_{N x}\right| \cdot\left(\left\|e_{N}\right\|\left\|e_{N x}\right\|+\left\|\eta_{N}\right\|\left\|e_{N x}\right\|\right) \\
& +|b| \sup _{x \in[0,1]}\left|e_{N}\right| \cdot\left\|e_{N}+\eta_{N}\right\|\left\|u_{x x}+u_{N x x}\right\| \\
\leq & |b| \sup _{x \in[0,1]}\left|u_{x}+u_{N x}\right| \cdot\left(\left\|e_{N}\right\|\left\|e_{N x}\right\|+\left\|\eta_{N}\right\|\left\|e_{N x}\right\|\right) \\
& +C|b|\left\|e_{N x}\right\| \cdot\left\|e_{N}+\eta_{N}\right\|\left\|u_{x x}+u_{N x x}\right\| \\
\leq & \left(c_{7}+c_{5}\right)|b|\left(\left\|e_{N x}\right\|^{2}+\frac{1}{2}\left\|e_{N}\right\|^{2}+\frac{1}{2}\left\|\eta_{N}\right\|^{2}\right) \\
& +2 C\left(c_{3}+c_{8}\right)|b|\left(\varepsilon\left\|e_{N x}\right\|^{2}+\frac{1}{2 \varepsilon}\left\|e_{N}\right\|^{2}+\frac{1}{2 \varepsilon}\left\|\eta_{N}\right\|^{2}\right) \\
\leq & \left(c_{7}+c_{5}\right)|b|\left(\varepsilon\left\|e_{N x x}\right\|^{2}+\left(\frac{1}{2}+\frac{1}{4 \varepsilon}\right)\left\|e_{N}\right\|^{2}+\frac{1}{2}\left\|\eta_{N}\right\|^{2}\right) \\
& +2 C\left(c_{3}+c_{8}\right)|b|\left(\varepsilon\left\|e_{N x x}\right\|^{2}+\left(\frac{1}{2 \varepsilon}+\frac{\varepsilon}{4}\right)\left\|e_{N}\right\|^{2}+\frac{1}{2 \varepsilon}\left\|\eta_{N}\right\|^{2}\right)
\end{aligned}
$$

where $\varepsilon \in \mathbb{R}^{+}$is a constant. Summing up, we get

$$
\begin{aligned}
& \frac{d}{d t}\left\|e_{N}\right\|^{2}+2\left(k-\left[\left(c_{5}+c_{7}\right)|b|+2 C\left(c_{3}+c_{8}\right)|b|\right] \varepsilon\right)\left\|e_{N x x}\right\|^{2} \\
& \quad \leq 4 k\left\|e_{N x}\right\|^{2}+2 c_{9}\left\|e_{N}\right\|^{2}+2 c_{10}\left\|\eta_{N}\right\|^{2} \\
& \quad=-4 k\left(e_{N}, e_{N x x}\right)+2 c_{9}\left\|e_{N}\right\|^{2}+2 c_{10}\left\|\eta_{N}\right\|^{2} \\
& \quad \leq \varepsilon\left\|e_{N x x}\right\|^{2}+\left(\frac{4 k^{2}}{\varepsilon}+2 c_{9}\right)\left\|e_{N}\right\|^{2}+2 c_{10}\left\|\eta_{N}\right\|^{2}
\end{aligned}
$$


where

$$
\begin{aligned}
& c_{9}=|\gamma|+\frac{3}{2}\left(c_{4}^{2}+c_{4} c_{6}+c_{6}^{2}\right)+\left(\frac{1}{2}+\frac{1}{4 \varepsilon}\right)\left(c_{5}+c_{7}\right)|b|+\left(\frac{1}{\varepsilon}+\frac{\varepsilon}{2}\right) C\left(c_{3}+c_{8}\right)|b|, \\
& c_{10}=\frac{1}{2}\left(c_{4}^{2}+c_{4} c_{6}+c_{6}^{2}\right)+\frac{1}{2}\left(c_{7}+c_{5}\right)|b|+\frac{1}{\varepsilon} C\left(c_{3}+c_{8}\right)|b| .
\end{aligned}
$$

From Theorem 1.1 and (B2), we have

$$
\left\|\eta_{N}\right\| \leq c N^{-2}\left\|u_{x x}\right\| \leq c_{11}\left(k, a, b, T,\left\|u_{0}\right\|_{H^{2}}\right) N^{-2} .
$$

Then a simple calculation shows that

$$
\begin{aligned}
& \frac{d}{d t}\left\|e_{N}\right\|^{2}+\left[2\left(k-\left[\left(c_{5}+c_{7}\right)|b|+2 C\left(c_{3}+c_{8}\right)|b|\right] \varepsilon\right)-\varepsilon\right]\left\|e_{N x x}\right\|^{2} \\
& \quad \leq\left(\frac{4 k^{2}}{\varepsilon}+2 c_{9}\right)\left\|e_{N}\right\|^{2}+2 c_{10}\left\|\eta_{N}\right\|^{2} \leq\left(\frac{4 k^{2}}{\varepsilon}+2 c_{9}\right)\left\|e_{N}\right\|^{2}+2 c_{10} c_{11}^{2} N^{-4},
\end{aligned}
$$

where $\varepsilon$ is small enough, it satisfies $2\left(k-\left[\left(c_{5}+c_{7}\right)|b|+2 C\left(c_{3}+c_{8}\right)|b|\right] \varepsilon\right)-\varepsilon>0$. Therefore, by Gronwall's inequality, we deduce that

$$
\left\|e_{N}\right\|^{2} \leq e^{\left(\frac{2}{\varepsilon}+2 c_{9}\right) T}\left\|e_{N}(0)\right\|^{2}+\frac{c_{10} c_{11}^{2}}{\left(1+c_{9}\right) \varepsilon} N^{-4} .
$$

Hence, the proof is completed.

\section{Fully discrete scheme}

In this section, we set up a full-discretization scheme for problem (2)-(4) and consider the fully discrete scheme which implies the pointwise boundedness of the solution.

Let $\Delta t$ be the time-step. The full-discretization spectral method for problem (2)-(4) is read as: find $u_{N}^{j} \in S_{N}(j=0,1,2, \ldots, N)$ such that for any $v_{N} \in S_{N}$, the following holds:

$$
\begin{aligned}
& \left(\frac{u_{N}^{j+1}-u_{N}^{j}}{\Delta t}, v_{N}\right)+k\left(\bar{u}_{N x x}^{j+\frac{1}{2}}, v_{N x x}\right)-2 k\left(\bar{u}_{N x}^{j+\frac{1}{2}}, v_{N x}\right) \\
& \quad+a\left(\bar{u}_{N}^{j+\frac{1}{2}}, v_{N}\right)+b\left(\left(\left(_{u_{N x}^{j+\frac{1}{2}}}^{j}\right)^{2}, v_{N}\right)+\left(\left(\bar{u}_{N}^{j+\frac{1}{2}}\right)^{3}, v_{N}\right)=0,\right.
\end{aligned}
$$

with $u_{N}(0)=P_{N} u_{0}$, where $\bar{u}_{N}^{j+\frac{1}{2}}=\frac{1}{2}\left(u_{N}^{j}+u_{N}^{j+1}\right)$.

The solution $u_{N}^{j}$ has the following property.

Lemma 3.1 Assume that $u_{0} \in H_{E}^{2}(0,1)$ and $b^{2} \leq 8 k$. Suppose that $u_{N}^{j}$ is a solution of problem (31), then there exist positive constants $c_{12}, c_{13}, c_{14}, c_{15}, c_{16}$ depending only on $k, a, b, T$ and $\left\|u_{0}\right\|_{H^{2}}$ such that

$$
\left\|u_{N}^{j}\right\| \leq c_{12}, \quad\left\|u_{N x}^{j}\right\| \leq c_{13}, \quad\left\|u_{N x x}^{j}\right\| \leq c_{14} .
$$

Furthermore, we have

$$
\sup _{x \in[0,1]}\left|u_{N}^{j}\right| \leq c_{15}, \quad \sup _{x \in[0,1]}\left|u_{N x}^{j}\right| \leq c_{16} .
$$


Proof It can be proved the same as Lemmas 2.1-2.3. Since the proof is so easy, we omit it.

In the following, we analyze the error estimates between numerical solution $u_{N}^{j}$ and exact solution $u\left(t_{j}\right)$. According to the properties of the projection operator $P_{N}$, we only need to analyze the error between $P_{N} u\left(t_{j}\right)$ and $u_{N}^{j}$. Denoted by $u^{j}=u\left(t_{j}\right), e^{j}=P_{N} u^{j}-u_{N}^{j}$ and $\eta^{j}=u^{j}-P_{N} u^{j}$. Therefore

$$
u^{j}-u_{N}^{j}=\eta^{j}+e^{j}
$$

If no confusion occurs, we denote the average of the two instant errors $e^{n}$ and $e^{n+1}$ by $\bar{e}^{n+\frac{1}{2}}$, where $\bar{e}^{n+\frac{1}{2}}=\frac{e^{n}+e^{n+1}}{2}$. On the other hand, we let $\bar{\eta}^{j+\frac{1}{2}}=\frac{\eta^{j}+\eta^{j+1}}{2}$.

Firstly, we give the following error estimates for the full discretization scheme.

Lemma 3.2 For the instant errors $e^{j+1}$ and $e^{j}$, we have

$$
\begin{aligned}
\left\|e^{j+1}\right\|^{2} \leq & \left\|e^{j}\right\|^{2}+2 \Delta t\left(u_{t}\left(t_{j+\frac{1}{2}}\right)-\frac{u_{N}^{j+1}-u_{N}^{j}}{\Delta t}, \bar{e}^{j+\frac{1}{2}}\right) \\
& +\frac{1}{320}(\Delta t)^{4} \int_{t_{j}}^{t_{j+1}}\left\|u_{t t t}\right\|^{2} d t+\Delta t\left\|\bar{e}^{j+\frac{1}{2}}\right\|^{2} .
\end{aligned}
$$

Proof Applying Taylor's expansion about $t_{j+\frac{1}{2}}$, using Hölder's inequality, we can prove the lemma immediately. Since the proof is the same as [11], we omit it.

Taking the inner product of (2) with $\bar{e}^{-j+\frac{1}{2}}$, and letting $t=t_{j+\frac{1}{2}}$, we obtain

$$
\begin{aligned}
& \left(u_{t}^{j+\frac{1}{2}}, \bar{e}^{j+\frac{1}{2}}\right)+\left(k u_{x x}^{j+\frac{1}{2}}, \bar{e}_{x x}^{j+\frac{1}{2}}\right)-2 k\left(u_{x}^{j+\frac{1}{2}}, \bar{e}_{x}^{j+\frac{1}{2}}\right)+a\left(u^{j+\frac{1}{2}}, \bar{e}^{j+\frac{1}{2}}\right) \\
& \quad+b\left(\left(u_{x}^{j+\frac{1}{2}}\right)^{2}, \bar{e}^{j+\frac{1}{2}}\right)+\left(\left(u^{j+\frac{1}{2}}\right)^{3}, \bar{e}^{j+\frac{1}{2}}\right)=0 .
\end{aligned}
$$

Taking $v_{N}=\bar{e}^{n+\frac{1}{2}}$ in (31), we obtain

$$
\begin{aligned}
& \left(\frac{u_{N}^{j+1}-u_{N}^{j}}{\Delta t}, \bar{e}^{j+\frac{1}{2}}\right)+k\left(\bar{u}_{N x x}^{j+\frac{1}{2}}, e_{x x}^{j+\frac{1}{2}}\right)-2 k\left(\bar{u}_{N x}^{j+\frac{1}{2}}, \bar{e}_{x}^{j+\frac{1}{2}}\right)+a\left(\bar{u}_{N}^{j+\frac{1}{2}}, \bar{e}^{j+\frac{1}{2}}\right) \\
& +b\left(\left(\bar{u}_{N x}^{j+\frac{1}{2}}\right)^{2}, \bar{e}^{j+\frac{1}{2}}\right)+\left(\left(\bar{u}_{N}^{j+\frac{1}{2}}\right)^{3}, \bar{e}^{j+\frac{1}{2}}\right)=0 .
\end{aligned}
$$

Comparing the above two equations, we get

$$
\begin{aligned}
\left(u_{t}^{j+\frac{1}{2}}\right. & \left.-\frac{u_{N}^{j+1}-u_{N}^{j}}{\Delta t}, \bar{e}^{j+\frac{1}{2}}\right) \\
= & -k\left(u_{x x}^{j+\frac{1}{2}}-\bar{u}_{N x x}^{j+\frac{1}{2}}, \bar{e}_{x x}^{j+\frac{1}{2}}\right)+2 k\left(u_{x}^{j+\frac{1}{2}}-\bar{u}_{N x}^{j+\frac{1}{2}}, \bar{e}_{x}^{j+\frac{1}{2}}\right)-a\left(u^{j+\frac{1}{2}}-\bar{u}_{N}^{j+\frac{1}{2}}, \bar{e}^{j+\frac{1}{2}}\right) \\
& -b\left(\left(u_{x}^{j+\frac{1}{2}}\right)^{2}-\left(\bar{u}_{N x}^{j+\frac{1}{2}}\right)^{2}, \bar{e}^{j+\frac{1}{2}}\right)-\left(\left(u^{j+\frac{1}{2}}\right)^{3}-\left(\bar{u}_{N}^{j+\frac{1}{2}}\right)^{3}, \bar{e}^{j+\frac{1}{2}}\right) .
\end{aligned}
$$

So, we investigate the error estimates of the five items on the right-hand side of the previous equation. 
Lemma 3.3 Suppose that $u_{0} \in H_{E}^{2}(0,1)$ and $b^{2} \leq 8 k, u$ is the solution for problem (2)-(4) and $u_{N}^{j}$ is the solution for problem (31), then

$$
-k\left(u_{x x}^{j+\frac{1}{2}}-\bar{u}_{N x x}^{j+\frac{1}{2}}, \bar{e}_{x x}^{j+\frac{1}{2}}\right) \leq-\frac{k}{2}\left\|\bar{e}_{x x}^{j+\frac{1}{2}}\right\|^{2}+\frac{k(\Delta t)^{3}}{192} \int_{t_{j}}^{t_{j+1}}\left\|u_{x x t t}\right\|^{2} d t
$$

Proof Using Taylor's expansion, we obtain

$$
\begin{aligned}
& u^{j}=u^{j+\frac{1}{2}}-\frac{\Delta t}{2} u_{t}^{j+\frac{1}{2}}+\int_{t_{j}}^{t_{j+\frac{1}{2}}}\left(t-t_{j}\right) u_{t t} d t \\
& u^{j+1}=u^{j+\frac{1}{2}}+\frac{\Delta t}{2} u_{t}^{j+\frac{1}{2}}+\int_{t_{j+\frac{1}{2}}}^{t_{j+1}}\left(t_{j}-t\right) u_{t t} d t .
\end{aligned}
$$

Hence

$$
\frac{1}{2}\left(u^{j}+u^{j+1}\right)-u^{j+\frac{1}{2}}=\frac{1}{2}\left(\int_{t_{j}}^{t_{j+\frac{1}{2}}}\left(t-t_{j}\right) u_{t t} d t+\int_{t_{j+\frac{1}{2}}}^{t_{j+1}}\left(t_{j}-t\right) u_{t t} d t\right) .
$$

By Hölder's inequality, we have

$$
\begin{aligned}
\left\|u_{x x}^{j+\frac{1}{2}}-\frac{1}{2}\left(u_{x x}^{j}+u_{x x}^{j+1}\right)\right\|^{2} & =\frac{1}{4}\left\|\left(\int_{t_{j}}^{t_{j+\frac{1}{2}}}\left(t-t_{j}\right) u_{t t} d t+\int_{t_{j+\frac{1}{2}}}^{t_{j+1}}\left(t_{j}-t\right) u_{t t} d t\right)_{x x}\right\|^{2} \\
& \leq \frac{(\Delta t)^{3}}{96} \int_{t_{j}}^{t_{j+1}}\left\|u_{x x t t}\right\|^{2} d t .
\end{aligned}
$$

Noticing that $\left(\bar{\eta}_{x x}^{j+\frac{1}{2}}, \bar{e}_{x x}^{j+\frac{1}{2}}\right)=0$. Therefore

$$
\begin{aligned}
-\left(u_{x x}^{j+\frac{1}{2}}-\bar{u}_{N x x}^{j+\frac{1}{2}}, \bar{e}_{x x}^{j+\frac{1}{2}}\right) & =-\left(u_{x x}^{j+\frac{1}{2}}-\frac{u_{x x}^{j}+u_{x x}^{j+1}}{2}, \bar{e}_{x x}^{j+\frac{1}{2}}\right)-\left(\frac{u_{x x}^{j+1}+u_{x x}^{j}}{2}-\frac{u_{N x x}^{j+1}+u_{N x x}^{j}}{2}, \bar{e}_{x x}^{j+\frac{1}{2}}\right) \\
& \leq\left\|u_{x x}^{j+\frac{1}{2}}-\frac{u_{x x}^{j}+u_{x x}^{j+1}}{2}\right\|\left\|\bar{e}_{x x}^{j+\frac{1}{2}}\right\|-\left(\bar{\eta}_{x x}^{j+\frac{1}{2}}+\bar{e}_{x x}^{j+\frac{1}{2}}, \bar{e}_{x x}^{j+\frac{1}{2}}\right) \\
& \leq\left(\frac{(\Delta t)^{3}}{96} \int_{t_{j}}^{t_{j+1}}\left\|u_{x x t t}\right\|^{2} d t\right)^{\frac{1}{2}}\left\|\bar{e}_{x x}^{j+\frac{1}{2}}\right\|-\left(\bar{\eta}_{x x}^{j+\frac{1}{2}}, \bar{e}_{x x}^{j+\frac{1}{2}}\right)-\left\|\bar{e}_{x x}^{j+\frac{1}{2}}\right\|^{2} \\
& \leq \frac{(\Delta t)^{3}}{192} \int_{t_{j}}^{t_{j+1}}\left\|u_{x x t t}\right\|^{2} d t-\frac{1}{2}\left\|\bar{e}_{x x}^{j+\frac{1}{2}}\right\|^{2} .
\end{aligned}
$$

Then Lemma 3.3 is proved.

Lemma 3.4 Suppose that $u_{0} \in H_{E}^{2}(0,1)$ and $b^{2} \leq 8 k, u$ is the solution for problem (2)-(4) and $u_{N}^{j}$ is the solution for problem (31), then

$$
\begin{aligned}
& 2 k\left(u_{x}^{j+\frac{1}{2}}-\bar{u}_{N x}^{j+\frac{1}{2}}, \bar{e}_{x}^{j+\frac{1}{2}}\right) \\
& \quad \leq \frac{k}{16}\left\|\bar{e}_{x x}^{j+\frac{1}{2}}\right\|^{2}+192 k\left\|\bar{e}^{j+\frac{1}{2}}\right\|^{2}+192 k c_{11} N^{-4}+\frac{k(\Delta t)^{3}}{2} \int_{t_{j}}^{t_{j+1}}\left\|u_{t t}\right\|^{2} d t,
\end{aligned}
$$

where $c_{11}$ is the same constant as (28). 
Proof Noticing that $\left\|\bar{\eta}^{j+\frac{1}{2}}\right\| \leq c N^{-2}$. Hence

$$
\begin{aligned}
& 2\left(u_{x}^{j+\frac{1}{2}}-\bar{u}_{N x}^{j+\frac{1}{2}}, \bar{e}_{x}^{j+\frac{1}{2}}\right) \\
& \quad=-2\left(u^{j+\frac{1}{2}}-\bar{u}_{N}^{j+\frac{1}{2}}, \bar{e}_{x x}^{j+\frac{1}{2}}\right) \\
& \quad=-2\left(u^{j+\frac{1}{2}}-\bar{u}^{j+\frac{1}{2}}, \bar{e}_{x x}^{j+\frac{1}{2}}\right)-2\left(\frac{u^{j+1}+u^{j}}{2}-\frac{u_{N}^{j+1}+u_{N}^{j}}{2}, \bar{e}_{x x}^{j+\frac{1}{2}}\right) \\
& \quad \leq 2\left\|u^{j+\frac{1}{2}}-\frac{u^{j+1}+u^{j}}{2}\right\|\left\|\bar{e}_{x x}^{j+\frac{1}{2}}\right\|+2\left\|\frac{u^{j+1}+u^{j}}{2}-\frac{u_{N}^{j+1}+u_{N}^{j}}{2}\right\|\left\|\bar{e}_{x x}^{j+\frac{1}{2}}\right\| \\
& \quad \leq 2\left\|\int_{t_{j}}^{t^{j+\frac{1}{2}}}\left(t-t_{j}\right) u_{t t} d t+\int_{t_{j+\frac{1}{2}}}^{t_{j+1}}\left(t_{j+1}-t\right) u_{t t} d t\right\|\left\|\bar{e}_{x x}^{j+\frac{1}{2}}\right\|+2\left\|\bar{e}^{j+\frac{1}{2}}+\bar{\eta}^{j+\frac{1}{2}}\right\|\left\|\bar{e}_{x x}^{j+\frac{1}{2}}\right\| \\
& \quad \leq 2\left(\frac{(\Delta t)^{3}}{96} \int_{t_{j}}^{t_{j+1}}\left\|u_{t t}\right\|^{2} d t\right)^{\frac{1}{2}}\left\|\bar{e}_{x x}^{j+\frac{1}{2}}\right\|+4\left\|\bar{e}^{j+\frac{1}{2}}\right\|\left\|\bar{e}_{x x}^{j+\frac{1}{2}}\right\|+4\left\|\bar{\eta}^{j+\frac{1}{2}}\right\|\left\|\bar{e}_{x x}^{j+\frac{1}{2}}\right\| \\
& \quad \leq 3 \varepsilon\left\|e_{x x}^{j+\frac{1}{2}}\right\|^{2}+\frac{4}{\varepsilon}\left(\left\|e^{j+\frac{1}{2}}\right\|^{2}+c_{11} N^{-4}\right)+\frac{(\Delta t)^{3}}{96 \varepsilon} \int_{t_{j}}^{t_{j+1}}\left\|u_{t t}\right\|^{2} d t .
\end{aligned}
$$

In the above inequality, setting $\varepsilon=\frac{1}{48}$, we get the conclusion.

Lemma 3.5 Suppose that $u_{0} \in H_{E}^{2}(0,1)$ and $b^{2} \leq 8 k, u$ is the solution for problem (2)-(4) and $u_{N}^{j}$ is the solution for problem (31), then

$$
\begin{aligned}
& -a\left(u^{j+\frac{1}{2}}-\bar{u}_{N}^{j+\frac{1}{2}}, \bar{e}^{j+\frac{1}{2}}\right) \\
& \quad \leq 4|a|\left\|e^{j+\frac{1}{2}}\right\|^{2}+|a| c_{11} N^{-4}+\frac{|a|(\Delta t)^{3}}{384} \int_{t_{j}}^{t_{j+1}}\left\|u_{t t}\right\|^{2} d t
\end{aligned}
$$

where $c_{11}$ is the same constant as (28).

Proof We have

$$
\begin{aligned}
& -a\left(u^{j+\frac{1}{2}}-\bar{u}_{N}^{j+\frac{1}{2}}, \bar{e}^{j+\frac{1}{2}}\right) \\
& =-a\left(u^{j+\frac{1}{2}}-\frac{u^{j+1}+u^{j}}{2}, \bar{e}^{j+\frac{1}{2}}\right)+a\left(\frac{u^{j+1}-u^{j}}{2}-\bar{u}_{N}^{j+\frac{1}{2}}, \bar{e}^{j+\frac{1}{2}}\right) \\
& \quad \leq|a|\left\|u^{j+\frac{1}{2}}-\frac{u^{j+1}+u^{j}}{2}\right\|\left\|\bar{e}^{j+\frac{1}{2}}\right\|+|a|\left\|\frac{u^{j+1}-u^{j}}{2}-\bar{u}_{N}^{j+\frac{1}{2}}\right\|\left\|\bar{e}^{j+\frac{1}{2}}\right\| \\
& \quad \leq|a|\left\|\int_{t_{j}}^{t_{j+\frac{1}{2}}}\left(t-t_{j}\right) u_{t t} d t+\int_{t_{j+\frac{1}{2}}}^{t_{j+1}}\left(t_{j+1}-t\right) u_{t t} d t\right\|\left\|\bar{e}^{j+\frac{1}{2}}\right\|+|a|\left\|\bar{e}^{j+\frac{1}{2}}+\bar{\eta}^{j+\frac{1}{2}}\right\|\left\|\bar{e}^{j+\frac{1}{2}}\right\| \\
& \quad \leq|a|\left(\frac{(\Delta t)^{3}}{96} \int_{t_{j}}^{t_{j+1}}\left\|u_{t t}\right\|^{2} d t\right)^{\frac{1}{2}}\left\|\bar{e}^{j+\frac{1}{2}}\right\|+2|a|\left(\left\|\bar{e}^{j+\frac{1}{2}}\right\|^{2}+\left\|\bar{\eta}^{j+\frac{1}{2}}\right\|\left\|\bar{e}^{j+\frac{1}{2}}\right\|\right) \\
& \quad \leq 4|a|\left\|\bar{e}^{j+\frac{1}{2}}\right\|^{2}+|a| c_{11} N^{-4}+\frac{|a|(\Delta t)^{3}}{384} \int_{t_{j}}^{t_{j+1}}\left\|u_{t t}\right\|^{2} d t .
\end{aligned}
$$

Then Lemma 3.5 is proved. 
Zhao et al. Advances in Difference Equations 2013, 2013:156

Page 13 of 19

Lemma 3.6 Suppose that $u_{0} \in H_{E}^{2}(0,1)$ and $b^{2} \leq 8 k$, $u$ is the solution for problem (2)-(4) and $u_{N}^{j}$ is the solution for problem (31), then

$$
\begin{aligned}
& -b\left(\left(u_{x}^{j+\frac{1}{2}}\right)^{2}-\left(\bar{u}_{N x}^{j+\frac{1}{2}}\right)^{2}, \bar{e}^{j+\frac{1}{2}}\right) \\
& \quad \leq \frac{k}{16}\left\|\bar{e}_{x x}^{j+\frac{1}{2}}\right\|^{2}+\frac{c_{7}|b|(\Delta t)^{3}}{96} \int_{t_{j}}^{t_{j+1}}\left\|u_{x t t}\right\|^{2} d t+c_{17} c_{11} N^{-4}+c_{18}\left\|\bar{e}^{j+\frac{1}{2}}\right\|^{2}
\end{aligned}
$$

where $C \in \mathbb{R}^{+}, c_{17}=c_{7}|b|+c_{16}|b|+C|b|\left(c_{8}+c_{14}\right)$ and $c_{18}=c_{7}|b|+c_{17}+\frac{8 c_{17}}{k}$.

Proof Notice that

$$
\sup _{x \in[0,1]}\left|u_{x}(x, t)\right| \leq c_{7}, \quad\left\|u_{x x}(x, t)\right\|^{2} \leq c_{8}, \quad\left\|u_{N x x}^{j}\right\| \leq c_{14}, \quad \sup _{x \in[0,1]}\left|u_{N x}^{j}\right| \leq c_{16} .
$$

Hence

$$
\begin{aligned}
-b\left(\left(u_{x}^{j+\frac{1}{2}}\right)^{2}-\left(\bar{u}_{N x}^{j+\frac{1}{2}}\right)^{2}, \bar{e}^{j+\frac{1}{2}}\right) & -b\left(\left(u_{x}^{j+\frac{1}{2}}+\frac{u_{x}^{j+1}+u_{x}^{j}}{2}\right)\left(u_{x}^{j+\frac{1}{2}}-\frac{u_{x}^{j+1}+u_{x}^{j}}{2}\right), \bar{e}^{j+\frac{1}{2}}\right) \\
= & b\left(\left(\frac{u_{x}^{j+1}+u_{x}^{j}}{2}+\bar{u}_{N x}^{j+\frac{1}{2}}\right)\left(\frac{u_{x}^{j+1}+u_{x}^{j}}{2}-\bar{u}_{N x}^{j+\frac{1}{2}}\right), \bar{e}^{j+\frac{1}{2}}\right) \\
= & -b\left(\left(u_{x}^{j+\frac{1}{2}}+\frac{u_{x}^{j+1}+u_{x}^{j}}{2}\right)\left(u_{x}^{j+\frac{1}{2}}-\frac{u_{x}^{j+1}+u_{x}^{j}}{2}\right), \bar{e}^{j+\frac{1}{2}}\right) \\
& +b\left(\left(\frac{u_{x}^{j+1}+u_{x}^{j}}{2}+\bar{u}_{N x}^{j+\frac{1}{2}}\right)\left(\frac{u^{j+1}+u^{j}}{2}-\frac{u_{N}^{j+1}+u_{N}^{j}}{2}\right), \bar{e}_{x}^{j+\frac{1}{2}}\right) \\
& +b\left(\left(\frac{u_{x x}^{j+1}+u_{x x}^{j}}{2}+\frac{u_{N x x}^{j+1}+u_{N x x}^{j}}{2}\right)\left(\frac{u^{j+1}+u^{j}}{2}-\frac{u_{N}^{j+1}+u_{N}^{j}}{2}\right), \bar{e}^{j+\frac{1}{2}}\right) .
\end{aligned}
$$

We have used the method of integration by parts in (33). Then

$$
\begin{aligned}
& -b\left(\left(u_{x}^{j+\frac{1}{2}}\right)^{2}-\left(\bar{u}_{N x}^{j+\frac{1}{2}}\right)^{2}, \bar{e}^{j+\frac{1}{2}}\right) \\
& \leq|b| \sup _{x \in[0,1]}\left|u_{x}^{j+\frac{1}{2}}+\frac{u_{x}^{j+1}+u_{x}^{j}}{2}\right| \cdot\left\|u_{x}^{j+\frac{1}{2}}-\frac{u_{x}^{j+1}+u_{x}^{j}}{2}\right\|\left\|\bar{e}^{j+\frac{1}{2}}\right\| \\
& \quad+|b| \sup _{x \in[0,1]}\left|\frac{u_{x}^{j+1}+u_{x}^{j}}{2}+\bar{u}_{N x}^{j+\frac{1}{2}}\right| \cdot\left\|\frac{u^{j+1}+u^{j}}{2}-\frac{u_{N}^{j+1}+u_{N}^{j}}{2}\right\|\left\|e_{x}^{j+\frac{1}{2}}\right\| \\
& \quad+|b| \sup _{x \in[0,1]}\left|e^{j+\frac{1}{2}}\right| \cdot\left\|\frac{u_{x x}^{j+1}+u_{x x}^{j}}{2}+\frac{u_{N x x}^{j+1}+u_{N x x}^{j}}{2}\right\|\left\|\frac{u^{j+1}+u^{j}}{2}-\frac{u_{N}^{j+1}+u_{N}^{j}}{2}\right\| \\
& \leq 2 c_{7}|b|\left\|\int_{t_{j}}^{t_{j+\frac{1}{2}}}\left(t-t_{j}\right) u_{x t t} d t+\int_{t_{j+\frac{1}{2}}}^{t_{j+1}}\left(t_{j+1}-t\right) u_{x t t} d t\right\|\left\|\bar{e}^{j+\frac{1}{2}}\right\| \\
& \quad+\left(c_{7}|b|+c_{16}|b|+C|b|\left(c_{8}+c_{14}\right)\right)\left\|e^{j+\frac{1}{2}}+\bar{\eta}^{j+\frac{1}{2}}\right\|\left\|\bar{e}_{x}^{j+\frac{1}{2}}\right\| \\
& \leq c_{7}|b| \frac{(\Delta t)^{3}}{96} \int_{t_{j}}^{t_{j+1}}\left\|u_{x t t}\right\|^{2} d t+c_{7}|b|\left\|\bar{e}^{j+\frac{1}{2}}\right\|^{2}+\varepsilon\left\|\bar{e}_{x x}^{j+\frac{1}{2}}\right\|^{2}
\end{aligned}
$$




$$
\begin{aligned}
& +c_{17}\left(\left\|e^{j+\frac{1}{2}}\right\|^{2}+\left\|\bar{\eta}^{j+\frac{1}{2}}\right\|^{2}\right)+\frac{c_{17}}{2 \varepsilon}\left\|e^{j+\frac{1}{2}}\right\|^{2} \\
\leq & \varepsilon\left\|e_{x x}^{j+\frac{1}{2}}\right\|^{2}+\frac{c_{7}|b|(\Delta t)^{3}}{96} \int_{t_{j}}^{t_{j+1}}\left\|u_{x t t}\right\|^{2} d t+\left(c_{7}|b|+c_{17}+\frac{c_{17}}{2 \varepsilon}\right)\left\|e^{j+\frac{1}{2}}\right\|^{2}+c_{17} c_{11} N^{-4} .
\end{aligned}
$$

Setting $\varepsilon=\frac{k}{16}$ in the above inequality, we obtain the conclusion.

Lemma 3.7 Suppose that $u_{0} \in H_{E}^{2}(0,1)$ and $b^{2} \leq 8 k, u$ is the solution for problem (2)-(4) and $u_{N}^{j}$ is the solution for problem (31), then

$$
-\left(\left(u^{j+\frac{1}{2}}\right)^{3}-\left(\bar{u}_{N}^{j+\frac{1}{2}}\right)^{3}, \bar{e}^{j+\frac{1}{2}}\right) \leq \frac{(\Delta t)^{3}}{96} \int_{t_{j}}^{t_{j+1}}\left\|u_{t t}\right\|^{2} d t+c_{19}\left\|\bar{e}^{j+\frac{1}{2}}\right\|^{2}+c_{20} c_{11} N^{-4},
$$

where $c_{19}=\frac{9 c_{6}^{2}}{4}+3\left(c_{6}^{2}+c_{6} c_{12}+c_{12}^{2}\right), c_{20}=c_{6}^{2}+c_{6} c_{12}+c_{12}^{2}$.

Proof Notice that

$$
\sup _{x \in[0,1]}\left|u^{j}\right| \leq c_{6}, \quad \sup _{x \in[0,1]}\left|u_{N}^{j}\right| \leq c_{12} .
$$

Hence

$$
\begin{aligned}
- & \left(\left(u^{j+\frac{1}{2}}\right)^{3}-\left(\bar{u}_{N}^{j+\frac{1}{2}}\right)^{3}, \bar{e}^{j+\frac{1}{2}}\right) \\
= & -\left(\left(u^{j+\frac{1}{2}}\right)^{3}-\left(\frac{u^{j+1}+u^{j}}{2}\right)^{3}, \bar{e}^{j+\frac{1}{2}}\right)-\left(\left(\frac{u^{j+1}+u^{j}}{2}\right)^{3}-\left(\bar{u}_{N}^{j+\frac{1}{2}}\right)^{3}, \bar{e}^{j+\frac{1}{2}}\right) \\
\leq & \sup _{x \in[0,1]} \mid\left(u^{j+\frac{1}{2}}\right)^{2}+u^{j+\frac{1}{2}} \frac{u^{j+1}+u^{j}}{2}+\left(\frac{u^{j+1}+u^{j}}{2}\right)^{2}\|\| u^{j+\frac{1}{2}}-\frac{u^{j+1}+u^{j}}{2}\|\| e^{j+\frac{1}{2}} \| \\
& +\sup _{x \in[0,1]} \mid\left(\frac{u^{j+1}+u^{j}}{2}\right)^{2}+\bar{u}_{N}^{j+\frac{1}{2}} \frac{u^{j+1}+u^{j}}{2}+\left(\bar{u}_{N}^{j+\frac{1}{2}}\right)^{2}\|\| \frac{u^{j+1}+u^{j}}{2}-\bar{u}_{N}^{j+\frac{1}{2}}\|\| \bar{e}^{j+\frac{1}{2}} \| \\
\leq & 3 c_{6}^{2}\left\|\int_{t_{j}}^{t_{j+\frac{1}{2}}}\left(t-t_{j}\right) u_{t t} d t+\int_{t_{j+\frac{1}{2}}}^{t_{j+1}}\left(t_{j+1}-t\right) u_{t t} d t\right\|\left\|\bar{e}^{j+\frac{1}{2}}\right\| \\
& +\left(c_{6}^{2}+c_{6} c_{12}+c_{12}^{2}\right)\left\|\bar{e}^{j+\frac{1}{2}}+\bar{\eta}^{j+\frac{1}{2}}\right\|\left\|\bar{e}^{j+\frac{1}{2}}\right\| \\
\leq & \frac{(\Delta t)^{3}}{96} \int_{t_{j}}^{t_{j+1}}\left\|u_{t t}\right\|^{2} d t+\left(\frac{9 c_{6}^{2}}{4}+3\left(c_{6}^{2}+c_{6} c_{12}+c_{12}^{2}\right)\right)\left\|e^{j+\frac{1}{2}}\right\|^{2} \\
& +\left(c_{6}^{2}+c_{6} c_{12}+c_{12}^{2}\right)\left\|\bar{\eta}^{j+\frac{1}{2}}\right\|^{2} \\
\leq & \frac{(\Delta t)^{3}}{96} \int_{t_{j}}^{t_{j+1}}\left\|u_{t t}\right\|^{2} d t+c_{19}\left\|\bar{e}^{j+\frac{1}{2}}\right\|^{2}+c_{20} c_{11} N^{-4} .
\end{aligned}
$$

Then Lemma 3.7 is proved.

Now, we obtain the following theorem.

Theorem 3.1 Suppose that $u_{0} \in H_{E}^{2}(0,1)$ and $b^{2} \leq 8 k, u(x, t)$ is the solution for problem (2)-(4) satisfying

$$
u \in L^{\infty}\left(0, T ; H^{2}(0,1)\right), \quad u_{t t} \in L^{2}\left(0, T ; H^{2}(0,1)\right), \quad u_{t t t} \in L^{2}\left(0, T ; L^{2}(0,1)\right) .
$$


Suppose further that $u_{N}^{j}$ is the solution for problem (31). Then if $\Delta t$ is sufficiently small, there exist positive constants $c_{21}$ depending on $k, a, b, T,\left\|u_{0}\right\|_{H^{2}}$ and $c_{22}$ depending on $k$, $a, b, T,\left\|u_{0}\right\|_{H^{2}}, \int_{0}^{T}\left\|u_{t t}\right\|_{H^{2}}^{2} d t$ and $\int_{0}^{T}\left\|u_{t t t}\right\|^{2} d t$ such that, for $j=0,1,2, \ldots, N$,

$$
\left\|e^{j+1}\right\| \leq c_{21}\left(N^{-2}+\left\|e^{0}\right\|\right)+c_{22}(\Delta t)^{2} .
$$

Proof By Lemmas 3.2-3.7, we obtain

$$
\begin{aligned}
\left\|e^{j+1}\right\|^{2} \leq & \left\|e^{j}\right\|^{2}+\Delta t c_{23}\left(\left\|e^{j+1}\right\|^{2}+\left\|e^{j}\right\|^{2}+N^{-4}\right) \\
& +(\Delta t)^{4} c_{24} \int_{t_{j}}^{t_{j+1}}\left(\left\|u_{t t}\right\|^{2}+\left\|u_{x t t}\right\|^{2}+\left\|u_{x x t t}\right\|^{2}+\left\|u_{t t t}\right\|^{2}\right) d t,
\end{aligned}
$$

where $c_{23}$ and $c_{24}$ are positive constants depending only on $k, a, b, T$ and $\left\|u_{0}\right\|_{H^{2}}$. For $\Delta t$ being sufficiently small such that $c_{24} \Delta t \leq \frac{1}{2}$, setting $c_{25}=2\left(c_{23}+c_{24}\right)$, we get

$$
\left\|e^{j+1}\right\|^{2} \leq\left(1+c_{25} \Delta t\right)\left\|e^{j}\right\|^{2}+c_{25}\left(\Delta t N^{-4}+(\Delta t)^{4} B^{j}\right)
$$

where

$$
B^{j}=\int_{t_{j}}^{t_{j+1}}\left(\left\|u_{t t}\right\|^{2}+\left\|u_{x t t}\right\|^{2}+\left\|u_{x x t t}\right\|^{2}+\left\|u_{t t t}\right\|^{2}\right) d t
$$

Using Gronwall's inequality for the discrete form, we have

$$
\left\|e^{j+1}\right\|^{2} \leq e^{c_{25}(j+1) \Delta t}\left(\left\|e^{0}\right\|^{2}+c_{25}\left(j \Delta t N^{-4}+(\Delta t)^{4} \sum_{i=0}^{j} B^{i}\right)\right) .
$$

Direct computation shows that

$$
\sum_{i=0}^{j} B^{i} \leq \int_{0}^{t_{j+1}}\left(\left\|u_{t t}\right\|^{2}+\left\|u_{x t t}\right\|^{2}+\left\|u_{x x t t}\right\|^{2}+\left\|u_{t t t}\right\|^{2}\right) d t .
$$

Thus, Theorem 3.1 is proved.

Furthermore, we have the following theorem.

Theorem 3.2 Suppose that $u_{0} \in H_{E}^{2}(0,1)$ and $b^{2} \leq 8 k, u(x, t)$ is the solution for problem (2)-(4) satisfying

$$
u \in L^{\infty}\left(0, T ; H^{2}(0,1)\right), \quad u_{t t} \in L^{2}\left(0, T ; H^{2}(0,1)\right), \quad u_{t t t} \in L^{2}\left(0, T ; L^{2}(0,1)\right) .
$$

Suppose further that $u_{N}^{j} \in S_{N}(j=0,1,2, \ldots)$ is the solution for problem (31) and the initial value $u_{N}^{0}$ satisfies $\left\|e^{0}\right\|=\left\|P_{N} u_{0}-u_{N}^{0}\right\| \leq c N^{-2}\left\|u_{x x}\right\|$. Then there exist positive constants $c^{\prime}$ depending on $k, a, b, T,\left\|u_{0}\right\|_{H^{2}}$ and $c^{\prime \prime}$ depending on $k, a, b, T,\left\|u_{0}\right\|_{H^{2}}, \int_{0}^{T}\left\|u_{t t}\right\|_{H^{2}}^{2} d t$, $\int_{0}^{T}\left\|u_{t t t}\right\|^{2} d t$ such that

$$
\left\|u\left(x, t_{j}\right)-u_{N}^{j}\right\| \leq c^{\prime} N^{-2}+c^{\prime \prime}(\Delta t)^{2}, \quad j=0,1,2, \ldots, N .
$$




\section{Numerical results}

In this section, using the spectral method described in (31), we carry out some numerical computations to illustrate out results in previous section. The full-discretization spectral method is read as: For $v_{l}=\sin l \pi x, l=1, \ldots, N$, find

$$
u_{N}^{n}=\sum_{i=1}^{N} \alpha_{i}^{n} \sin i \pi x, \quad i=1, \ldots, N,
$$

such that (31) holds.

Noticing that under the inner product $(\cdot, \cdot),\{\sin i \pi x, i=1,2, \ldots\}$ is the system of orthogonal functions, then

$$
\int_{0}^{1} \sin i_{1} \pi x \cdot \sin i_{2} \pi x d x= \begin{cases}0, & i_{1} \neq i_{2}, \\ \frac{1}{2}, & i_{1}=i_{2} .\end{cases}
$$

Therefore, the terms of (31) are

$$
\begin{aligned}
& \left(\frac{u_{N}^{j+1}-u_{N}^{j}}{\Delta t}, v_{l}\right)=\frac{\alpha_{l}^{j+1}-\alpha_{l}^{j}}{2 \Delta t}, \\
& k\left(\bar{u}_{N x x}^{j+\frac{1}{2}}, v_{l x x}\right)=\frac{k}{2}\left(u_{N x x}^{j+1}+u_{N x x}^{j}, v_{l x x}\right)=\frac{k}{4}(l \pi)^{4}\left(\alpha_{l}^{j+1}+\alpha_{l}^{j}\right), \\
& -2 k\left(\bar{u}_{N x}^{j+\frac{1}{2}}, v_{l x}\right)=k\left(u_{N}^{j+1}+u_{N}^{j}, v_{l x x}\right)=-\frac{k}{2}(l \pi)^{2}\left(\alpha_{l}^{j+1}+\alpha_{l}^{j}\right), \\
& a\left(\bar{u}_{N}^{j+\frac{1}{2}}, v_{l}\right)=\frac{a}{2}\left(u_{N}^{j+1}+u_{N}^{j}, v_{l}\right)=\frac{a}{4}\left(\alpha_{l}^{j+1}+\alpha_{l}^{j}\right), \\
& b\left(\left(\bar{u}_{N x}^{j+\frac{1}{2}}\right)^{2}, v_{l}\right)=\frac{b}{4}\left(\left(u_{N x}^{j+1}+u_{N x}^{j}\right)^{2}, v_{l}\right) \\
& =\frac{b}{4} \sum_{p_{1}, p_{2}=1}^{N} p_{1} p_{2} \pi\left(\alpha_{p_{1}}^{j+1} \alpha_{p_{2}}^{j+1}+\alpha_{p_{1}}^{j} \alpha_{p_{2}}^{j}+2 \alpha_{p_{1}}^{j+1} \alpha_{p_{2}}^{j}\right) \xi_{p_{1} p_{2} l}
\end{aligned}
$$

and

$$
\begin{aligned}
& \left(\left(\bar{u}_{N}^{j+\frac{1}{2}}\right)^{3}, v_{l}\right) \\
& \quad=\frac{1}{8} \sum_{p_{1}, p_{2}, p_{3}=1}^{N}\left(\alpha_{p_{1}}^{j+1} \alpha_{p_{2}}^{j+1} \alpha_{p_{3}}^{j+1}+3 \alpha_{p_{1}}^{j+1} \alpha_{p_{2}}^{j+1} \alpha_{p_{3}}^{j}+3 \alpha_{p_{1}}^{j+1} \alpha_{p_{2}}^{j} \alpha_{p_{3}}^{j}+\alpha_{p_{1}}^{j} \alpha_{p_{2}}^{j} \alpha_{p_{3}}^{j}\right) \eta_{p_{1} p_{2} p_{3} l},
\end{aligned}
$$

where

$$
\begin{aligned}
& \xi_{p_{1} p_{2} l}=\int_{0}^{1} \cos p_{1} \pi x \cdot \cos p_{2} \pi x \cdot \sin l \pi x d x \\
& \eta_{p_{1} p_{2} p_{3} l}=\int_{0}^{1} \sin p_{1} \pi x \cdot \sin p_{2} \pi x \cdot \sin p_{3} \pi x \cdot \sin l \pi x d x .
\end{aligned}
$$

Thus, (31) can be transformed as

$$
\frac{\alpha_{l}^{j+1}-\alpha_{l}^{j}}{2 \Delta t}+\left(\frac{k}{4}(l \pi)^{4}-\frac{k}{2}(l \pi)^{2}+\frac{a}{4}\right)\left(\alpha_{l}^{j+1}+\alpha_{l}^{j}\right)+\frac{b}{4} \rho_{l}+\frac{1}{8} \sigma_{l}=0,
$$


where $l=1, \ldots, N$, and

$$
\begin{aligned}
& \rho_{l}=\sum_{p_{1}, p_{2}=1}^{N} p_{1} p_{2} \pi^{2}\left(\alpha_{p_{1}}^{j+1} \alpha_{p_{2}}^{j+1}+\alpha_{p_{1}}^{j} \alpha_{p_{2}}^{j}+2 \alpha_{p_{1}}^{j+1} \alpha_{p_{2}}^{j}\right) \xi_{p_{1} p_{2} l}, \\
& \sigma_{l}=\sum_{p_{1}, p_{2}, p_{3}=1}^{N}\left(\alpha_{p_{1}}^{j+1} \alpha_{p_{2}}^{j+1} \alpha_{p_{3}}^{j+1}+3 \alpha_{p_{1}}^{j+1} \alpha_{p_{2}}^{j+1} \alpha_{p_{3}}^{j}+3 \alpha_{p_{1}}^{j+1} \alpha_{p_{2}}^{j} \alpha_{p_{3}}^{j}+\alpha_{p_{1}}^{j} \alpha_{p_{2}}^{j} \alpha_{p_{3}}^{j}\right) \eta_{p_{1} p_{2} p_{3} l} .
\end{aligned}
$$

If $\alpha_{k}^{n}(k=1,2, \ldots, N)$ is known, there exists an $N$ variable nonlinear system of equations for $\alpha_{l}^{j+1}(l=1,2, \ldots, N)$ which can be seen as

$$
\mathbf{F}\left(\alpha^{j+1}\right)=\left(\begin{array}{c}
f_{1}\left(\alpha^{j+1}\right) \\
f_{2}\left(\alpha^{j+1}\right) \\
\vdots \\
f_{N}\left(\alpha^{j+1}\right)
\end{array}\right)=\mathbf{0}
$$

We use the simple Newton method to seek the solutions. Initialization yields

$$
\alpha_{(0)}^{j+1}=\left(\alpha_{1}^{j}, \alpha_{2}^{j}, \ldots, \alpha_{N}^{j}\right)^{T}
$$

The iterative formulation is as follows:

$$
\left.\begin{array}{l}
\alpha_{(k+1)}^{j+1}=\alpha_{(k)}^{j+1}+\Delta \alpha_{(k)}^{j+1}, \\
\mathbf{F}^{\prime}\left(\alpha_{(0)}^{j+1}\right) \cdot \Delta \alpha_{(k)}^{j+1}+\mathbf{F}\left(\alpha_{(k)}^{j+1}\right)=0, \quad k=0,1,2, \ldots,
\end{array}\right\}
$$

where $\mathbf{F}^{\prime}\left(\alpha_{(0)}^{j+1}\right)$ is the $N \times N$ order Jacobi matrix for $\mathbf{F}\left(\alpha^{j+1}\right)$ when $\alpha^{j+1}=\alpha_{(0)}^{j+1}$,

$$
\mathbf{F}^{\prime}\left(\alpha_{(0)}^{j+1}\right)=\left(\begin{array}{ccc}
\partial_{1} f_{1}\left(\alpha_{(0)}^{j+1}\right), & \ldots, & \partial_{N} f_{1}\left(\alpha_{(0)}^{j+1}\right) \\
\vdots & \ddots & \vdots \\
\partial_{1} f_{N}\left(\alpha_{(0)}^{j+1}\right), & \ldots, & \partial_{N} f_{N}\left(\alpha_{(0)}^{j+1}\right)
\end{array}\right)
$$

Give accuracy $\epsilon>0$, when $\left\|\alpha_{(k+1)}^{j+1}-\alpha_{(k)}^{j+1}\right\|<\epsilon$, stop the iteration, $\alpha^{j+1} \approx \alpha_{(k+1)}^{j+1}$.

As an example, we choose $k=2, a=1, b=1, u_{0}=(1-x)^{5} x^{5}, \Delta t=0.0005, N=32$, and get the solution which evolves from $t=0$ to $t=0.025$ (cf. Figure 1 ).

Figure 1 Expanded property of solution when $N=32, \Delta t=0.0005$.

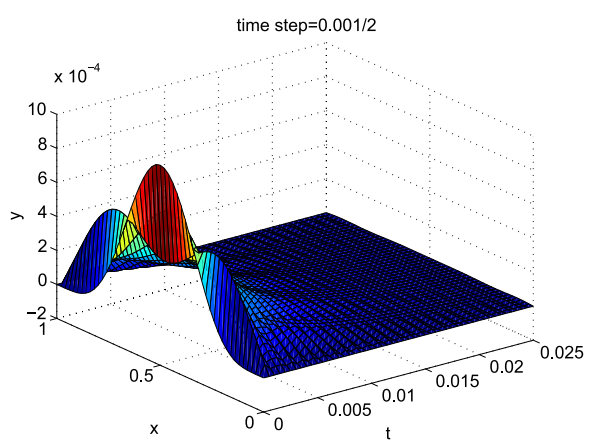


Table 1 Errors of different time steps at $t=0.1$

\begin{tabular}{lll}
\hline $\boldsymbol{\Delta t}$ & $\operatorname{err}(\mathbf{0 . 1}, \boldsymbol{\Delta t})$ & $\frac{\operatorname{err}(\mathbf{0 . 1}, \boldsymbol{\Delta} \boldsymbol{t})}{(\boldsymbol{\Delta} \boldsymbol{t})^{\mathbf{2}}}$ \\
\hline 0.001 & $1.6808 \times 10^{-6}$ & 1.6808 \\
$0.001 \times \frac{1}{2}$ & $2.2439 \times 10^{-7}$ & 0.8976 \\
$0.001 \times \frac{1}{4}$ & $2.6068 \times 10^{-8}$ & 0.4171 \\
$0.001 \times \frac{1}{8}$ & $2.8494 \times 10^{-9}$ & 0.1824 \\
$0.001 \times \frac{1}{16}$ & $2.8754 \times 10^{-10}$ & 0.0736 \\
\hline
\end{tabular}

Table 2 Errors of different basic function numbers at $t=0.1$

\begin{tabular}{lll}
\hline $\boldsymbol{N}$ & $\operatorname{err}\left(\mathbf{0 . 1}, \boldsymbol{\Delta} \boldsymbol{t}_{\mathbf{0}}\right)$ & $\frac{\operatorname{err}\left(\mathbf{0 . 1}, \boldsymbol{\Delta} \boldsymbol{t}_{\mathbf{0}}\right)}{\boldsymbol{N}^{-\mathbf{2}}}$ \\
\hline 24 & $2.28708 \times 10^{-10}$ & $1.32 \times 10^{-7}$ \\
28 & $1.3588 \times 10^{-10}$ & $1.07 \times 10^{-7}$ \\
32 & $7.32182 \times 10^{-11}$ & $7.50 \times 10^{-8}$ \\
36 & $3.87087 \times 10^{-11}$ & $5.02 \times 10^{-8}$ \\
40 & $2.03256 \times 10^{-11}$ & $3.25 \times 10^{-8}$ \\
\hline
\end{tabular}

Now, we consider the variation of error. Since there is no exact solution for (2)-(4) known to us, we make a comparison between the solution of (31) on coarse meshes and a fine mesh.

Choose $\Delta t=0.001,0.001 \times \frac{1}{2}, 0.001 \times \frac{1}{4}, 0.001 \times \frac{1}{8}, 0.001 \times \frac{1}{10}, 0.001 \times \frac{1}{16}$, respectively, to solve (31). Set $u_{N}^{\min }(x, 0.1)$ as the solution for $\Delta t_{\min }=0.001 \times \frac{1}{32}$. Denote

$$
\operatorname{err}(0.1, \Delta t)=\left(\int_{0}^{1}\left(u_{N}^{k}(x, 0.1)-u_{N}^{\min }(x, 0.1)\right)^{2} d x\right)^{\frac{1}{2}}, \quad k=1,2, \ldots, 6
$$

Then the error is showed in Table 1 at $t=0.1$.

On the other hand, choose $N=24,28,32,36,40, \Delta t_{0}=0.001 \times \frac{1}{16}$, respectively, to solve (31). Then the error is showed in Table 2 at $t=0.1$.

It is easy to see that the third column $\frac{\operatorname{err}(0.1, \Delta t)}{(\Delta t)^{2}}$ of Table 1 is monotone decreasing along with the time step's waning, the third column $\frac{\operatorname{err}\left(0.1, \Delta t_{0}\right)}{N^{-2}}$ of Table 2 is monotone decreasing along with $N$ 's magnifying. Hence, we can find positive constants $C_{1}=1.6808, C_{2}=1.32 \times$ $10^{-7}$ such that

$$
\frac{\operatorname{err}(0.1, \Delta t)}{(\Delta t)^{2}} \leq C, \quad k=1,2, \ldots, 6
$$

and

$$
\frac{\operatorname{err}\left(0.1, \Delta t_{0}\right)}{N^{-2}} \leq C, \quad N=24,28,32,36,40
$$

Thus, the order of error estimates is $O\left((\Delta t)^{2}+N^{-2}\right)$ proved in Theorem 3.2.

The authors declare that they have no competing interests. 


\section{Author details}

${ }^{1}$ College of Mathematics, Jilin University, Changchun, 130012, China. ${ }^{2}$ Institute of Software, Chinese Academy of Sciences, Beijing, 100190, China.

\section{Acknowledgements}

This work is supported by the Graduate Innovation Fund of Jilin University (Project 20121059). The authors would like to express their deep thanks for the referee's valuable suggestions about the revision and improvement of the manuscript.

Received: 28 December 2012 Accepted: 16 May 2013 Published: 4 June 2013

\section{References}

1. Doelman, A, Standstede, B, Scheel, A, Schneider, G: Propagation of hexagonal patterns near onset. Eur. J. Appl. Math. $14,85-110$ (2003)

2. Song, L, Zhang, Y, Ma, T: Global attractor of a modified Swift-Hohenberg equation in $H^{k}$ space. Nonlinear Anal. 72 183-191 (2010)

3. Swift, J, Hohenberg, PC: Hydrodynamics fluctuations at the convective instability. Phys. Rev. A 15, 319-328 (1977)

4. La Quey, RE, Mahajan, PH, Rutherford, PH, Tang, WM: Nonlinear saturation of the trapped-ion mode. Phys. Rev. Lett. 34, 391-394 (1975)

5. Shlang, T, Sivashinsky, GL: Irregular flow of a liquid film down a vertical column. J. Phys. France 43, 459-466 (1982)

6. Kuramoto, Y: Diffusion-induced chaos in reaction systems. Prog. Theor. Phys. Suppl. 64, 346-347 (1978)

7. Polat, M: Global attractor for a modified Swift-Hohenberg equation. Comput. Math. Appl. 57, 62-66 (2009)

8. Sivashinsky, GL: Nonlinear analysis of hydrodynamic instability in laminar flames. Acta Astron. 4, 1177-1206 (1977)

9. Lega, J, Moloney, JV, Newell, AC: Swift-Hohenberg equation for lasers. Phys. Rev. Lett. 73, 2978-2981 (1994)

10. Peletier, LA, Rottschäfer, V: Large time behavior of solution of the Swift-Hohenberg equation. C. R. Math. Acad. Sci. Paris, Sér. I 336, 225-230 (2003)

11. Chai, S, Zou, Y, Gong, C: Spectral method for a class of Cahn-Hilliard equation with nonconstant mobility. Commun. Math. Res. 25, 9-18 (2009)

12. He, Y, Liu, Y: Stability and convergence of the spectral Galerkin method for the Cahn-Hilliard equation. Numer Methods Partial Differ. Equ. 24, 1485-1500 (2008)

13. $Y e, X$, Cheng, $X$ : The Fourier spectral method for the Cahn-Hilliard equation. Appl. Math. Comput. 171, $345-357$ (2005)

14. Yin, $L, X u, Y$, Huang, $M$ : Convergence and optimal error estimation of a pseudo-spectral method for a nonlinear Boussinesq equation. J. Jilin Univ. Sci. 42, 35-42 (2004)

15. Canuto, C, Hussaini, MY, Quarteroni, A, Zang, TA: Spectral Methods in Fluid Dynamics. Springer, New York (1988)

16. Xiang, X: The Numerical Analysis for Spectral Methods. Science Press, Beijing (2000) (in Chinese)

doi:10.1186/1687-1847-2013-156

Cite this article as: Zhao et al.: Fourier spectral method for the modified Swift-Hohenberg equation. Advances in Difference Equations 2013 2013:156.

\section{Submit your manuscript to a SpringerOpen ${ }^{\ominus}$ journal and benefit from:}

- Convenient online submission

- Rigorous peer review

Immediate publication on acceptance

- Open access: articles freely available online

- High visibility within the field

- Retaining the copyright to your article 